\title{
MPC of constrained discrete-time linear periodic systems - A framework for asynchronous control: Strong feasibility, stability and optimality via periodic invariance ${ }^{\text {th }}$
}

\author{
Ravi Gondhalekar ${ }^{\mathrm{a}, *}$, Colin N. Jones ${ }^{\mathrm{b}}$ \\ ${ }^{a}$ Department of Mechanical Engineering and Frontier Research Base for Global Young Researchers, Graduate School of Engineering, Osaka University, 2-1 Yamadaoka, Suita-shi, \\ Osaka, 565-0871, Japan \\ ${ }^{\mathrm{b}}$ Automatic Control Laboratory, Department of Electrical Engineering, Swiss Federal Institute of Technology in Zurich (ETHZ), ETL I 28, Physikstrasse 3, 8092 Zurich, Switzerland
}

\section{A R T I C L E I N F O}

\section{Article history:}

Available online 9 December 2010

\section{Keywords:}

Model predictive control

Constrained control

Set invariance

Linear periodic systems

Asynchronous control

\begin{abstract}
A B S T R A C T
State-feedback model predictive control (MPC) of discrete-time linear periodic systems with timedependent state and input dimensions is considered. The states and inputs are subject to periodically time-dependent, hard, convex, polyhedral constraints. First, periodic controlled and positively invariant sets are characterized, and a method to determine the maximum periodic controlled and positively invariant sets is derived. The proposed periodic controlled invariant sets are then employed in the design of least-restrictive strongly feasible reference-tracking MPC problems. The proposed periodic positively invariant sets are employed in combination with well-known results on optimal unconstrained periodic linear-quadratic regulation (LQR) to yield constrained periodic LQR control laws that are stabilizing and optimal. One motivation for systems with time-dependent dimensions is efficient control law synthesis for discrete-time systems with asynchronous inputs, for which a novel modeling framework resulting in low dimensional models is proposed. The presented methods are applied to a multirate nano-positioning system.
\end{abstract}

(C) 2010 Elsevier Ltd. All rights reserved.

\section{Introduction}

State-feedback MPC theory for constrained discrete-time linear time-invariant (LTI) systems is well established (Maciejowski, 2002; Mayne, Rawlings, Rao, \& Scokaert, 2000; Qin \& Badgwell, 2003). In this paper well-known LTI-MPC theory is generalized to perform MPC of constrained discrete-time linear periodic systems-useful extensions of LTI systems (Bittanti \& Colaneri, 2009; Lovera \& Varga, 2005; Meyer \& Burrus, 1975; Varga, 2007). Of particular interest are methods that accommodate periodically time-dependent state and input dimensions. Key to the proposed periodic MPC approach is the incorporation of periodic set invariance concepts, and the application of solutions to reverse periodic discrete-time algebraic Riccati equations (RP-DAREs), which were considered for the optimal LQR of unconstrained linear

\footnotetext{
the material in this paper was partially presented at the 48th IEEE CDC, December 16-18, 2009, Shanghai, China. This paper was recommended for publication in revised form by Associate Editor Tongwen Chen under the direction of Editor Ian R. Petersen. This work was supported in part by the Program of Promotion of Environmental Improvement to Enhance Young Researchers' Independence under the Special Coordination Funds for Promoting Science and Technology, Japan Ministry of Education, Culture, Sports, Science and Technology.

* Corresponding author. Tel.: +81 066879 7337; fax: +81 0668797335.

E-mail addresses: ravi.gondhalekar@wakate.frc.eng.osaka-u.ac.jp (R. Gondhalekar), cjones@ee.ethz.ch (C.N. Jones).
}

periodic systems with time-dependent dimensions in Chu, Fan, Lin, and Wang (2004) and Varga (2007, 2008). Invariant sets (Blanchini, 1999) are indispensable in constrained MPC (Mayne et al., 2000), as they yield tools to engineer the closed-loop behavior of MPC control laws with a finite prediction horizon. In the proposed periodic MPC methods maximum periodic controlled invariant sets are employed to least-restrictively enforce strong feasibility (Definition 14) of reference-tracking MPC problems (Theorem 16), for any prediction horizon length. This is crucial in practice, where short prediction horizons are often required (Qin \& Badgwell, 2003). Furthermore, for performing constrained periodic LQR, periodic positively invariant sets are used in conjunction with solutions to RP-DAREs to enforce a sufficient condition for closed-loop stability (Theorem 19), and to facilitate selecting the prediction horizon length such that the MPC problem is guaranteed to yield the optimal solution (Theorem 20). The proposed periodic MPC methods are generalizations of LTI-MPC methods, both in the sense that the MPC concepts are extended from the LTI to the periodic setting, but importantly also in that when the periodic system has period length one they reduce to the well-known LTI-MPC methods. Thus the MPC results of this paper will seem very natural to readers familiar with Mayne et al. (2000).

Suitably general periodic set invariance has not been characterized. An appropriate definition for (maximum) periodic positively (Definition 3) and controlled (Definition 4) invariant sets is proposed here, and a method for their determination based on cyclic 
shift-invariant lifting (Bittanti \& Colaneri, 2000; Flamm, 1991; Park \& Verriest, 1989) and invariant set methods for LTI systems (Blanchini, 1999; Dória \& Hennet, 1999; Gilbert \& Tan, 1991) is presented. Very closely related polyhedral maximum periodic controlled invariant sets were considered in Blanchini and Ukovich (1993). Ellipsoidal periodic positively invariant sets were considered in Böhm, Yu, and Allgöwer (2009). This paper considers polyhedral sets, which are more appropriate for systems with polyhedral constraints, and furthermore considers maximum invariant sets, which are crucial for reducing conservativeness in MPC. Neither Blanchini and Ukovich (1993) nor Böhm, Yu et al. (2009) considered time-dependent dimensions. Conceptually distinct periodic invariance for linear parameter varying systems was considered in e.g. Lee and Kouvaritakis (2006), and is not applicable here as it does not account for periodically time-dependent dynamics and constraints.

MPC of discrete-time linear/nonlinear periodic systems was tackled in Böhm, Raff, Reble, and Allgöwer (2009), Böhm, Yu et al. (2009), Kern, Böhm, Findeisen, and Allgöwer (2009), Kim, Lee, and Kwon (2000) and Lee, Natarajan, and Lee (2001)/Böhm, Yu et al. (2009) and Reble, Böhm, and Allgöwer (2009). Only unconstrained systems were treated in Kim et al. (2000). State constraints were not accommodated in Kern et al. (2009). In Lee et al. (2001) the predicted open-loop input trajectory is updated only once per period, introducing delays in responding to disturbances, plant-model mismatches and reference changes, the cumulative effects of which are collectively rejected only at integer multiples of the period length (see Section 6). This may cause severe performance degradation even at small disturbance/mismatch levels, and causes performance deterioration with increased period length at constant levels of disturbances/mismatches. Similar delays are encountered in Böhm, Raff et al.(2009), where predicted state-feedback control laws are recomputed once per period. The proposed methods respond at each step to disturbances, mismatches and reference changes. In Lee et al. (2001) the horizon length must be a multiple of the period length, whereas the proposed approach allows any horizon length, even one shorter than one period length. The methods of Böhm, Yu et al. (2009) and Reble et al. (2009) share key ingredients with the proposed Theorem 19 to enforce stability when performing constrained LQR. However, the use of ellipsoidal terminal constraint sets in Böhm, Yu et al. (2009) and Reble et al. (2009) generally leads to conservativeness, while in this paper the use of maximum periodic invariant sets avoids conservativeness. However, Böhm, Yu et al. (2009) and Reble et al. (2009) handle nonlinear systems, whereas the proposed methods are limited to linear systems. In Böhm, Raff et al. (2009), Böhm, Yu et al. (2009) and Reble et al. (2009) it was not considered how to least-restrictively enforce strong feasibility of reference-tracking MPC problems, or how to verify optimality when performing constrained LQR. Time-dependent dimensions were considered in Lee et al. (2001) but not in Böhm, Raff et al. (2009), Böhm, Yu et al. (2009), Kern et al. (2009), Kim et al. (2000) and Reble et al. (2009).

In this paper systems with time-dependent state and input dimensions are proposed as a succinct model of systems with asynchronous inputs, e.g. multirate inputs, where each input channel ${ }^{1}$ may have a unique update frequency, and multiplexed inputs, where input channels are updated in ordered sequence. Unconstrained multirate LTI systems were considered in e.g. Colaneri and De Nicolao (1995), Colaneri, Scattolini, and Schiavoni (1992), Longhi (1994), Sågfors, Toivonen, and Lennartson (2000), Scattolini and Schiavoni (1995) and Sezer and Šiljak (1990), constrained multiplexed LTI systems in Ling, Maciejowski, and Wu (2005) and Richards, Ling, and Maciejowski (2007). There the LTI

\footnotetext{
1 An input channel is one dimension $u_{[k]}$ of a multi-dimensional input vector $u$.
}

system is modeled by a linear periodic system with the timeinvariant state dimension extended by the input dimension. The extended states are used either to store actual inputs (Colaneri \& De Nicolao, 1995; Colaneri et al., 1992; Longhi, 1994; Sågfors et al., 2000; Sezer \& Šiljak, 1990), or to integrate the slew inputs (Ling et al., 2005; Richards et al., 2007; Scattolini \& Schiavoni, 1995), for future use. Crucially, all inputs are stored even if needless. Asynchronous inputs are modeled by either forcing to zero (Colaneri \& De Nicolao, 1995; Colaneri et al., 1992; Longhi, 1994; Scattolini \& Schiavoni, 1995; Sezer \& Šiljak, 1990), or eliminating (Ling et al., 2005; Richards et al., 2007; Sågfors et al., 2000), rows of the input distribution matrix associated with inputs that are not updated. The approach proposed here employs the latter method, which results in a lower, possibly time-dependent number of inputs. However, the proposed approach further exploits timedependent state dimensions to store only those inputs required later. This may yield significantly lower dimensional models, and is crucial for MPC and invariant set methods, where computational complexity is sensitive to both state and input dimension. Another motivation for time-dependent dimensions is that they are generally required for minimal periodic system realizations from input-output maps (Colaneri \& Longhi, 1995; Gohberg, Kaashoek, \& Lerer, 1992; Varga, 2004).

This paper presents rigorous treatment of preliminary results reported in Gondhalekar and Jones (2009).

Notation. The set of reals is denoted by $\mathbb{R}$, the set of nonnegative/positive integers by $\mathbb{N} / \mathbb{N}_{+}$, the set of consecutive nonnegative integers $\{j, \ldots, k\}$ by $\mathbb{N}_{j}^{k}$. For sets $\mathbb{A}, \mathbb{B}$ let $\mathbb{A} \backslash \mathbb{B}:=\{x \in$ $\mathbb{A} \mid x \notin \mathbb{B}\}$. Let $\bmod : \mathbb{N} \times \mathbb{N}_{+} \rightarrow \mathbb{N}, \bmod (i, j):=\min _{k \in \mathbb{N}}\{i-k j \mid i-$ $k j \geq 0\}$. Denote by $I_{n}$ the $n \times n$ identity matrix, by $0_{\{n, m\}}$ the $n \times m$ zero matrix, by 0 the zero matrix with appropriate dimension, by $\operatorname{diag}\left(a_{1}, \ldots, a_{n}\right)$ the block-diagonal matrix of $n$ elements $a_{i}$, by $a_{[j]}$ element $j$ of vector $a$, by $\rho(A)$ the spectral radius of matrix $A$. Denote by $A_{[:, \ell]}$ and $A_{[\ell,:]}$ the submatrices of matrix $A$ consisting of the columns and rows of $A$ with indices in set $\ell \subset \mathbb{N}_{+}$, respectively. A sequence of elements $x_{i} \in \mathbb{X} \forall i \in \mathbb{N}_{j}^{k}$ is denoted by $\left\{x_{i} \in \mathbb{X}\right\}_{i=j}^{k}$. Let $\psi_{(i, k)}$ denote the future value of $\psi$ at step $i+k$, as predicted from step $i$. Let $\psi_{i}:=\psi_{(i, 0)}$.

\section{Unconstrained periodic LQR}

Consider the discrete-time linear periodic system $x_{i+1}=A_{j} x_{i}+B_{j} u_{i}, \quad j:=\bmod (i, p)$

with system step $i \in \mathbb{N}$, period length $p \in \mathbb{N}_{+}$, inter-period step index $j \in \mathbb{N}_{0}^{p-1}$, state $x_{i} \in \mathbb{R}^{n_{j}}$ and input $u_{i} \in \mathbb{R}^{m_{j}}$. The following holds for all $j \in \mathbb{N}_{0}^{p-1}: A_{j} \in \mathbb{R}^{n_{\bmod (j+1, p)} \times n_{j}}, B_{j} \in \mathbb{R}^{n_{\bmod (j+1, p)} \times m_{j}}$, and $n_{j}, m_{j} \in \mathbb{N}_{+}$.

Remark 1. The presented methods are in concept applicable when $n_{j}=0$ or $m_{j}=0$ for some values of $j$. To avoid notational abuses or obfuscated notation only systems with strictly positive dimensions are explicitly considered. See Section 5 for an example where $m_{j}=0$.

Let $\left\{\mathrm{x}_{i} \in \mathbb{R}^{n_{j}}\right\}_{i=0}^{\infty}$ and $\left\{\mathrm{u}_{i} \in \mathbb{R}^{m_{j}}\right\}_{i=0}^{\infty}$ describe some reference state and input trajectory which should be tracked, respectively. For brevity let $\chi:=\left[x^{\top}, u^{\top}\right]^{\top}$ and $r:=\left[\mathrm{x}^{\top}, \mathrm{u}^{\top}\right]^{\top}$. For initial state $x_{i} \in \mathbb{R}^{n_{j}}$ the control objective is to minimize quadratic cost $V_{j}: \mathbb{R}^{n_{j}} \rightarrow \mathbb{R}$

$V_{j}\left(x_{i}\right):=\sum_{k=i}^{\infty}\left[\chi_{k}-r_{k}\right]^{\top} \Gamma_{\bmod (k, p)}\left[\chi_{k}-r_{k}\right]$,

with $\Gamma_{j}:=\left[\begin{array}{cc}Q_{j} & S_{j} \\ S_{j}^{\top} & R_{j}\end{array}\right] \succeq 0, Q_{j}=Q_{j}^{\top} \in \mathbb{R}^{n_{j} \times n_{j}}, R_{j}=R_{j}^{\top} \in \mathbb{R}^{m_{j} \times m_{j}}$, $S_{j} \in \mathbb{R}^{n_{j} \times m_{j}} \forall j \in \mathbb{N}_{0}^{p-1}$. Suppose the control objective is unconstrained periodic LQR: $r_{i}=0 \forall i \in \mathbb{N}$. The minimizer $V_{j}^{*}\left(x_{i}\right):=x_{i}^{\top} P_{j} x_{i}$ 
is achieved by periodic linear state-feedback $u_{k}=K_{\bmod (k, p)} x_{k} \forall k \in$ $\mathbb{N}_{i}^{\infty}, K_{j} \in \mathbb{R}^{m_{j} \times n_{j}} \forall j \in \mathbb{N}_{0}^{p-1}$ given by (3), where $\left\{P_{0}, \ldots, P_{p-1}\right\}, P_{j} \in$ $\mathbb{R}^{n_{j} \times n_{j}} \forall j \in \mathbb{N}_{0}^{p-1}$ is the unique, positive semi-definite, periodically stabilizing solution of RP-DARE (2). See Chu et al. (2004) and Varga $(2007,2008)$ for solution methods and conditions for the existence of solutions of RP-DAREs.

$$
\begin{aligned}
P_{j}= & Q_{j}+A_{j}^{\top} P_{\bmod (j+1, p)} A_{j}-\left(B_{j}^{\top} P_{\bmod (j+1, p)} A_{j}+S_{j}^{\top}\right)^{\top} \\
& \cdot\left[B_{j}^{\top} P_{\bmod (j+1, p)} B_{j}+R_{j}\right]^{-1} \cdot\left(B_{j}^{\top} P_{\bmod (j+1, p)} A_{j}+S_{j}^{\top}\right) \\
K_{j}:=- & {\left[B_{j}^{\top} P_{\bmod (j+1, p)} B_{j}+R_{j}\right]^{-1} \cdot\left(B_{j}^{\top} P_{\bmod (j+1, p)} A_{j}+S_{j}^{\top}\right) . }
\end{aligned}
$$

\section{Periodic set invariance}

In this section periodic set invariance (Blanchini, 1999; Blanchini \& Ukovich, 1993; Böhm, Yu et al., 2009) is characterized, and an approach for determining maximum periodic invariant sets is presented. The proposed sets are employed in Section 4 for designing constrained periodic MPC control laws with desirable properties. The sets are easily robustified against additive disturbances using standard methods (Blanchini, 1999; Dória \& Hennet, 1999). Robustness is ignored here for brevity. If $p=1$ then (1) is LTI and well-known methods to determine invariant sets may be applied (Blanchini, 1999; Dória \& Hennet, 1999; Gilbert \& Tan, 1991). For the definitions below to be correct make Assumption 2 .

\section{Assumption 2. $p \geq 2$.}

System (1) is required to satisfy polyhedral constraints $E_{j} x_{i}+G_{j} u_{i} \leq W_{j}$

with $E_{j} \in \mathbb{R}^{r_{j} \times n_{j}}, G_{j} \in \mathbb{R}^{r_{j} \times m_{j}}, W_{j} \in \mathbb{R}^{r_{j}}, r_{j} \in \mathbb{N}_{+} \forall j \in \mathbb{N}_{0}^{p-1}$. This paper is restricted to constraints (4) as these lead to tractable computational procedures.

Definition 3. A set $\left\{\mathbb{P}_{0}, \ldots, \mathbb{P}_{p-1}\right\}$ of sets $\mathbb{P}_{j} \subseteq \mathbb{R}^{n_{j}} \forall j \in \mathbb{N}_{0}^{p-1}$ is a periodic positively invariant set for system (1) subject to (4) under periodic linear state-feedback $u_{i}=K_{\bmod (i, p)} x_{i}, K_{j} \in \mathbb{R}^{m_{j} \times n_{j}} \forall j \in$ $\mathbb{N}_{0}^{p-1}$ if and only if:

$$
\begin{aligned}
& \left(A_{j}+B_{j} K_{j}\right) x \in \mathbb{P}_{\bmod (j+1, p)} \wedge\left(E_{j}+G_{j} K_{j}\right) x \leq W_{j} \\
& \forall x \in \mathbb{P}_{j} \forall j \in \mathbb{N}_{0}^{p-1} .
\end{aligned}
$$

The maximum periodic positively invariant set is the set $\left\{\mathbb{P}_{0}^{*}, \ldots\right.$, $\left.\mathbb{P}_{p-1}^{*}\right\}$ of sets $\mathbb{P}_{j}^{*} \subseteq \mathbb{R}^{n_{j}} \forall j \in \mathbb{N}_{0}^{p-1}$ as follows:

$$
\begin{aligned}
\mathbb{P}_{j}^{*}:= & \left\{x \in \mathbb{R}^{n_{j}} \mid x_{i+1}=\left(A_{k}+B_{k} K_{k}\right) x_{i} \wedge\left(E_{k}+G_{k} K_{k}\right) x_{i} \leq W_{k}\right. \\
& \left.\wedge k=\bmod (i, p) \forall i \in \mathbb{N}_{j}^{\infty}, x_{j}=x\right\} .
\end{aligned}
$$

Sets $\mathbb{P}_{j}^{*}$ constituting the maximum periodic positively invariant set contain each state $x_{k}$ such that applying periodic linear statefeedback $u_{i}=K_{\bmod (i, p)} x_{i} \forall i \in \mathbb{N}_{k}^{\infty}$ achieves constraint satisfaction indefinitely.

Definition 4. A set $\left\{\mathbb{C}_{0}, \ldots, \mathbb{C}_{p-1}\right\}$ of sets $\mathbb{C}_{j} \subseteq \mathbb{R}^{n_{j}} \forall j \in \mathbb{N}_{0}^{p-1}$ is a periodic controlled invariant set for system (1) subject to (4) if and only if:

$\forall j \in \mathbb{N}_{0}^{p-1} \forall x \in \mathbb{C}_{j} \exists u \in \mathbb{R}^{m_{j}}$ s.t.

$A_{j} x+B_{j} u \in \mathbb{C}_{\bmod (j+1, p)} \wedge E_{j} x+G_{j} u \leq W_{j}$.

The maximum periodic controlled invariant set is the set $\left\{\mathbb{C}_{0}^{*}, \ldots\right.$, $\left.\mathbb{C}_{p-1}^{*}\right\}$ of sets $\mathbb{C}_{j}^{*} \subseteq \mathbb{R}^{n_{j}} \forall j \in \mathbb{N}_{0}^{p-1}$ as follows:

$$
\begin{aligned}
\mathbb{C}_{j}^{*}:= & \left\{x \in \mathbb{R}^{n_{j}} \mid \exists\left\{u_{i} \in \mathbb{R}^{\left.m_{\bmod (i, p)}\right\}_{i=j}^{\infty} \text { s.t. }}\right.\right. \\
& x_{i+1}=A_{k} x_{i}+B_{k} u_{i} \wedge E_{k} x_{i}+G_{k} u_{i} \leq W_{k} \\
& \left.\wedge k=\bmod (i, p) \forall i \in \mathbb{N}_{j}^{\infty}, x_{j}=x\right\} .
\end{aligned}
$$

Sets $\mathbb{C}_{j}^{*}$ constituting the maximum periodic controlled invariant set contain each state such that there exists an infinite input trajectory such that applying this input trajectory achieves constraint satisfaction indefinitely.

We define the parameters

$$
\begin{aligned}
& \mathbf{A}:=\left[\begin{array}{cc}
0 & A_{p-1} \\
\operatorname{diag}\left(A_{0}, \ldots, A_{p-2}\right) & 0
\end{array}\right] \in \mathbb{R}^{\mathbf{n} \times \mathbf{n}} \\
& \mathbf{B}:=\left[\begin{array}{cc}
0 & B_{p-1} \\
\operatorname{diag}\left(B_{0}, \ldots, B_{p-2}\right) & 0
\end{array}\right] \in \mathbb{R}^{\mathbf{n} \times \mathbf{m}} \\
& \mathbf{K}:=\operatorname{diag}\left(K_{0}, \ldots, K_{p-1}\right) \in \mathbb{R}^{\mathbf{m} \times \mathbf{n}} \\
& \mathbf{E}:=\operatorname{diag}\left(E_{0}, \ldots, E_{p-1}\right) \in \mathbb{R}^{\mathbf{r} \times \mathbf{n}} \\
& \mathbf{G}:=\operatorname{diag}\left(G_{0}, \ldots, G_{p-1}\right) \in \mathbb{R}^{\mathbf{r} \times \mathbf{m}} \\
& \mathbf{W}:=\left[W_{0}^{\top}, \ldots, W_{p-1}^{\top}\right]^{\top} \in \mathbb{R}^{\mathbf{r}} \\
& \mathbf{n}:=\sum_{j=0}^{p-1} n_{j}, \quad \mathbf{m}:=\sum_{j=0}^{p-1} m_{j}, \quad \mathbf{r}:=\sum_{j=0}^{p-1} r_{j}
\end{aligned}
$$

and consider cyclic shift-invariant lifted LTI system (7) with $\mathbf{x} \in$ $\mathbb{R}^{\mathbf{n}}, \mathbf{u} \in \mathbb{R}^{\mathbf{m}}$ (Bittanti \& Colaneri, 2000; Flamm, 1991; Park \& Verriest, 1989):

$$
\mathbf{x}_{i+1}=\mathbf{A} \mathbf{x}_{i}+\mathbf{B} \mathbf{u}_{i}, \quad \mathbf{E} \mathbf{x}_{i}+\mathbf{G} \mathbf{u}_{i} \leq \mathbf{W} .
$$

Equivalent conditions to Eqs. (5) and (6) are Eqs. (8) and (9), respectively, where $\mathbb{P}:=\left(\mathbb{P}_{0} \times \cdots \times \mathbb{P}_{p-1}\right) \subseteq \mathbb{R}^{\mathbf{n}}$ and $\mathbb{C}:=$ $\left(\mathbb{C}_{0} \times \cdots \times \mathbb{C}_{p-1}\right) \subseteq \mathbb{R}^{\mathbf{n}}$.

$$
(\mathbf{A}+\mathbf{B K}) \mathbf{x} \in \mathbb{P} \wedge(\mathbf{E}+\mathbf{G K}) \mathbf{x} \leq \mathbf{W} \quad \forall \mathbf{x} \in \mathbb{P}
$$

$\forall \mathbf{x} \in \mathbb{C} \exists \mathbf{u} \in \mathbb{R}^{\mathbf{m}}$ s.t. $\mathbf{A x}+\mathbf{B u} \in \mathbb{C} \wedge \mathbf{E x}+\mathbf{G u} \leq \mathbf{W}$.

Maximum positively and controlled invariant sets $\mathbb{P}^{*} \subseteq \mathbb{R}^{\mathbf{n}}$ and $\mathbb{C}^{*} \subseteq \mathbb{R}^{\mathbf{n}}$ of cyclic shift-invariant lifted LTI system (7) are given by Eqs. (10) and (11), respectively (Blanchini, 1999).

$$
\begin{aligned}
\mathbb{P}^{*} & :=\left\{\mathbf{x}_{0} \in \mathbb{R}^{\mathbf{n}} \mid \mathbf{x}_{i+1}=(\mathbf{A}+\mathbf{B K}) \mathbf{x}_{i} \wedge(\mathbf{E}+\mathbf{G K}) \mathbf{x}_{i} \leq \mathbf{W} \forall i \in \mathbb{N}\right\} \\
\mathbb{C}^{*} & :=\left\{\mathbf{x}_{0} \in \mathbb{R}^{\mathbf{n}} \mid \exists\left\{\mathbf{u}_{i} \in \mathbb{R}^{\mathbf{m}}\right\}_{i=0}^{\infty}\right. \text { s.t. } \\
& \left.\mathbf{x}_{i+1}=\mathbf{A} \mathbf{x}_{i}+\mathbf{B} \mathbf{u}_{i} \wedge \mathbf{E} \mathbf{x}_{i}+\mathbf{G} \mathbf{u}_{i} \leq \mathbf{W} \forall i \in \mathbb{N}\right\} .
\end{aligned}
$$

Assumption 5. $\mathbf{W}>0$. (I.e. $W_{j}>0 \forall j \in \mathbb{N}_{0}^{p-1}$; the origin is within the interior of the constraints.)

Assumption 6. The pair $(\mathbf{A}, \mathbf{B})$ is stabilizable.

Lemma 7. The set $\mathbb{C}^{*}$ exists, contains the origin in its interior and is closed and convex.

Lemma 8. Suppose some periodic linear state-feedback gains $K_{j} \in$ $\mathbb{R}^{m_{j} \times n_{j}} \forall j \in \mathbb{N}_{0}^{p-1}$ stabilize system (1), i.e. K satisfies $\rho(\mathbf{A}+\mathbf{B K})<1$. Then the set $\mathbb{P}^{*}$ exists, contains the origin in its interior and is closed and convex.

The reader is referred to Blanchini (1999), Dória and Hennet (1999) and Gilbert and Tan (1991) for proofs and discussion of Lemmata 7 and 8.

Assumption 9. $\mathbb{P}^{*}$ and $\mathbb{C}^{*}$ are closed, convex polyhedra.

By Lemmata 7 and 8 , sets $\mathbb{P}^{*}$ and $\mathbb{C}^{*}$ are closed and convex. Thus either they are, or can be approximated arbitrarily closely by, closed and convex polyhedra. If Assumption 9 does not hold we suppose to have taken polyhedral under-approximations of $\mathbb{C}^{*}$ and $\mathbb{P}^{*}$ that are controlled and positively invariant, respectively. Under Assumption 9, $\mathbb{P}^{*}$ and $\mathbb{C}^{*}$ can straightforwardly be unlifted to yield maximum periodic invariant sets $\left\{\mathbb{P}_{0}^{*}, \ldots, \mathbb{P}_{p-1}^{*}\right\}$ and $\left\{\mathbb{C}_{0}^{*}, \ldots, \mathbb{C}_{p-1}^{*}\right\}$ such that $\mathbb{P}^{*}=\left(\mathbb{P}_{0}^{*} \times \cdots \times \mathbb{P}_{p-1}^{*}\right)$ and $\mathbb{C}^{*}=$ $\left(\mathbb{C}_{0}^{*} \times \cdots \times \mathbb{C}_{p-1}^{*}\right)$. 
Remark 10. Under Assumption 9 and by construction due to the unlifting, sets $\mathbb{P}_{j}^{*}$ and $\mathbb{C}_{j}^{*} \forall j \in \mathbb{N}_{0}^{p-1}$ are closed, convex polyhedra containing the origin in their interiors.

Remark 11. Cyclic shift-invariant LTI system (7) allows mathematical insight from the invariant set theory of LTI systems (Blanchini, 1999; Dória \& Hennet, 1999; Gilbert \& Tan, 1991) to be directly applied to periodic invariant sets, e.g. via Lemmata 7 and 8 . However, for the numerical determination of periodic invariant sets, employing the periodic system directly, without lifting, may result in shorter computation-times, especially when the period length $p$ is large. Maximum periodic controlled invariant sets with time-dependent dimensions can also be determined by applying the method of Blanchini and Ukovich (1993) with trivial modifications (details omitted), as is done in Section 6 .

Note that cyclic shift-invariant lifted LTI system (7) is employed nowhere in this paper outside of Section 3.

\section{MPC of linear periodic systems}

State-feedback periodic MPC control law synthesis for reference-tracking and periodic $L Q R$ is tackled next. The proposed methods could be robustified against additive disturbances using standard methods (Bemporad \& Morari, 1999; Goulart, Kerrigan, \& Maciejowski, 2006). Robustness is not explicitly included in the control law derivation as it is not a contribution of this paper, and for brevity.

For prediction horizon length $N \in \mathbb{N}_{+}$, MPC achieves closedloop control of system (1) subject to (4) by applying at each step $i$ the first input $u_{(i, 0)}$ of a predicted open-loop input trajectory $U_{i}:=\left[u_{(i, 0)}^{\top}, \ldots, u_{(i, N-1)}^{\top}\right]^{\top} \in \mathbb{R}^{v_{\bmod (i, p)}}, v_{j}:=\sum_{k=j}^{j+N-1} m_{\bmod (k, p)} \forall j \in$ $\mathbb{N}_{0}^{p-1}$. An optimal predicted open-loop input trajectory is determined by solving periodic MPC Problem 12 with cost matrices $T_{j} \in \mathbb{R}^{n_{j} \times n_{j}}, T_{j} \succeq 0, \Gamma_{j} \in \mathbb{R}^{\left(n_{j}+m_{j}\right) \times\left(n_{j}+m_{j}\right)}, \Gamma_{j} \succeq 0$ and terminal constraint sets $\mathbb{T}_{j} \subseteq \mathbb{R}^{n_{j}} \forall j \in \mathbb{N}_{0}^{p-1}$. The solution to MPC Problem 12 may not be unique. Parameters $N, T_{j}, \Gamma_{j}$ and $\mathbb{T}_{j}$ are the design parameters of MPC Problem 12. Note that prediction horizon length $N$ and system period length $p$ are independent.

Problem 12. Determine

$U_{i}^{*}\left(x_{i}\right):=\arg \min _{U_{i} \in \mathcal{U}_{j}\left(x_{i}\right)} J_{j}\left(x_{i}, U_{i}\right)$

subject to

$$
\begin{aligned}
& J_{j}\left(x_{i}, U_{i}\right):=\left[x_{(i, N)}-\mathrm{x}_{(i, N)}\right]^{\top} T_{\bmod (i+N, p)}\left[x_{(i, N)}-\mathrm{x}_{(i, N)}\right] \\
& +\sum_{k=0}^{N-1}\left[\chi_{(i, k)}-r_{(i, k)}\right]^{\top} \Gamma_{\bmod (i+k, p)}\left[\chi_{(i, k)}-r_{(i, k)}\right] \\
& u_{j}\left(x_{i}\right):=\left\{\begin{array}{l|l}
U_{i} \in \mathbb{R}^{v_{j}} & \begin{array}{l}
x_{(i, N)} \in \mathbb{T}_{\bmod (i+N, p)}, \\
E_{\bmod (i+k, p)} x_{(i, k)}+G_{\bmod (i+k, p)} u_{(i, k)} \\
\leq W_{\bmod (i+k, p)} \quad \forall k \in \mathbb{N}_{0}^{N-1}
\end{array}
\end{array}\right\} \\
& x_{(i, k+1)}=A_{\bmod (i+k, p)} x_{(i, k)}+B_{\bmod (i+k, p)} u_{(i, k)} \text {. }
\end{aligned}
$$

Remark 13. The exact choice of predicted reference values $r_{(i, k)}$ depends on the available information about the reference trajectory $\left\{r_{k}\right\}_{k=i}^{i+N}$ at time $i$ (see Section 6), and does not affect the results of this paper (see Remark 17).

Define sets $\mathbb{X}_{j}$ of feasible states as follows: $\mathbb{X}_{j}:=\left\{x \in \mathbb{R}^{n_{j}} \mid u_{j}(x)\right.$ $\neq \emptyset\} \forall j \in \mathbb{N}_{0}^{p-1}$. Note that sets $\mathbb{X}_{j}$ are independent of predicted reference values $r_{(i, k)}$ and prediction cost matrices $\Gamma_{j}$ and $T_{j}$.
Definition 14. MPC Problem 12 is strongly feasible if and only if from every feasible state $x_{k} \in \mathbb{X}_{\bmod (k, p)}$ the closed-loop state trajectory $\left\{x_{i}\right\}_{i=k}^{\infty}$ due to any sequence of feasible solutions $\left\{U_{i} \in\right.$ $\left.u_{\bmod (i, p)}\left(x_{i}\right)\right\}_{i=k}^{\infty}$ remains within the sets of feasible states.

Note that strongly feasible MPC problems are not guaranteed to yield stabilizing control laws.

Definition 15. Suppose a specific set of parameters $\left\{N, \mathbb{T}_{j}\right\}$ in MPC Problem 12 results in the sets $\hat{\mathbb{X}}_{j} \subseteq \mathbb{R}^{n_{j}} \forall j \in \mathbb{N}_{0}^{p-1}$ of feasible states. Then MPC Problem 12 with $\left\{N, \mathbb{T}_{j}\right\}$ is termed least-restrictive if and only if any other choice of parameters results in $\mathbb{X}_{j} \subseteq \hat{\mathbb{X}}_{j} \forall j \in \mathbb{N}_{0}^{p-1}$.

Theorem 16. If $\mathbb{T}_{j}=\mathbb{C}_{j}^{*} \forall j \in \mathbb{N}_{0}^{p-1}$ in MPC Problem 12, then MPC Problem 12 is a least-restrictive strongly feasible MPC problem.

Proof. Strong feasibility is enforced as follows. If at feasible state $x_{i}$ MPC Problem 12 determines any solution $U_{i} \in U_{j}\left(x_{i}\right)$ then $x_{(i, N)} \in \mathbb{C}_{\bmod (i+N, p)}^{*}$. At the next state $x_{i+1}=x_{(i, 1)}=A_{\bmod (i, p)} x_{i}+$ $B_{\bmod (i, p)} u_{(i, 0)}$ an admissible input trajectory up to but not including the final control move $u_{(i+1, N-1)}$ is given by the shifted solution of the step before: $u_{(i+1, k)}=u_{(i, k+1)} \forall k \in \mathbb{N}_{0}^{N-2}$. Applying this achieves $x_{(i+1, N-1)} \in \mathbb{C}_{\bmod (i+N, p)}^{*}$. The existence of an admissible final control move $u_{(i+1, N-1)}$ such that $x_{(i+1, N)} \in \mathbb{C}_{\bmod (i+N+1, p)}^{*}$ is guaranteed by the periodic controlled invariance property of Eq. (6). By induction this holds recursively. Least-restrictiveness results from the terminal constraint sets being the sets constituting the maximum periodic controlled invariant set. For all $j \in \mathbb{N}_{0}^{p-1}$ the sets $\mathbb{X}_{j}$ of feasible states are the $N$-step reach-sets to $\mathbb{T}_{\bmod (j+N, p)}$. If $\mathbb{T}_{j}=\mathbb{C}_{j}^{*} \forall j \in \mathbb{N}_{0}^{p-1}$ then $\mathbb{X}_{j}=\mathbb{C}_{j}^{*} \forall j \in \mathbb{N}_{0}^{p-1} \forall N \in \mathbb{N}_{+}$. By Definitions 4 and 14 there cannot exist a strongly feasible MPC problem with sets of feasible states which are strict supersets of the sets constituting the maximum periodic controlled invariant set. Therefore MPC Problem 12 is least-restrictive according to Definition 15.

Any non-maximum periodic controlled invariant set can be employed in Theorem 16 to enforce strong feasibility of MPC Problem 12, but may result in an MPC problem that is not leastrestrictive.

Remark 17. Theorem 16 is independent of reference trajectory $r_{i}$ and predicted references $r_{(i, k)}$. In contrast, Theorems 19 and 20 are developed specifically for periodic LQR to the origin: $r_{i}=0 \forall i \in \mathbb{N}$.

Assumption 18. $\Gamma_{j} \succ 0 \forall j \in \mathbb{N}_{0}^{p-1}$.

Assumption 18 states that stage cost matrices are positive definite. The solution to MPC Problem 12 is thereby unique. Less strict assumptions are possible but ignored here for brevity. Suppose the solution $\left\{P_{0}, \ldots, P_{p-1}\right\}$ of RP-DARE (2), periodic statefeedback gains $\left\{K_{0}, \ldots, K_{p-1}\right\}$ of (3), and any appropriate periodic positively invariant set $\left\{\mathbb{P}_{0}, \ldots, \mathbb{P}_{p-1}\right\}$ have been determined.

Theorem 19. Suppose $r_{i}=0 \forall i \in \mathbb{N}$. If $T_{j}=P_{j}, \mathbb{T}_{j}=\mathbb{P}_{j} \forall j \in$ $\mathbb{N}_{0}^{p-1}$ and $r_{(i, k)}=0 \forall(i, k) \in \mathbb{N} \times \mathbb{N}_{0}^{N}$ in MPC Problem 12, then MPC Problem 12 results in a stabilizing control law.

Proof. This follows closely the discussion of Mayne et al. (2000). Prediction cost $J_{\bmod (i, p)}\left(x_{i}, U_{i}^{*}\left(x_{i}\right)\right)$ is an upper bound of prediction cost $J_{\bmod (i+1, p)}\left(x_{i+1}, U_{i+1}^{*}\left(x_{i+1}\right)\right)$ at the next step. If $x_{i} \neq 0$ then the upper bound is strict. The prediction cost is monotonically decreasing and thus a Lyapunov function of the closed-loop system. Consider a feasible state $x_{i} \neq 0$. The solution $U_{i}^{*}\left(x_{i}\right)$ achieves $x_{(i, N)} \in \mathbb{P}_{\bmod (i+N, p)}$. At the next state $x_{i+1}=x_{(i, 1)} \mathrm{a}$ feasible solution $U_{i+1} \in \mathcal{U}_{\bmod (i+1, p)}\left(x_{i+1}\right)$ is given by $u_{(i+1, k)}=$ $u_{(i, k+1)} \forall k \in \mathbb{N}_{0}^{N-2}$ and $u_{(i+1, N-1)}=K_{\bmod (i+N, p)} x_{(i+1, N-1)}$. Thus $J_{\bmod (i+1, p)}\left(x_{i+1}, U_{i+1}^{*}\left(x_{i+1}\right)\right)-J_{\bmod (i, p)}\left(x_{i}, U_{i}^{*}\left(x_{i}\right)\right) \leq-\chi_{i}^{\top} \Gamma_{\bmod (i, p)} \chi_{i}$ $<0$ by Assumption 18 . 
Theorem 20. Suppose $r_{i}=0 \forall i \in \mathbb{N}$. If $T_{j}=P_{j}, \mathbb{T}_{j}=\mathbb{P}_{j} \forall j \in \mathbb{N}_{0}^{p-1}$ and $r_{(i, k)}=0 \forall(i, k) \in \mathbb{N} \times \mathbb{N}_{0}^{N}$ in MPC Problem 12, and iffurthermore solution $U_{i}^{*}\left(x_{i}\right)$ of MPC Problem 12 is such that the terminal constraint is not active (i.e. $\left.x_{(i, N)} \in \operatorname{interior}\left(\mathbb{C}_{\bmod (i+N, p)}\right)\right)$, then $U_{k}^{*}\left(x_{k}\right) \forall k \in \mathbb{N}_{i}^{\infty}$ is infinite-horizon optimal.

Proof. This follows closely the discussion of Mayne et al. (2000). Solution $U_{i}^{*}\left(x_{i}\right)$ is the first portion of the input trajectory that is determined in closed-loop. As the terminal constraint is not active the terminal cost incorporates the exact cost associated with the infinite-horizon prediction beyond prediction horizon $N$. Consider a feasible state $x_{i}$ and solution $U_{i}^{*}\left(x_{i}\right)$ such that $x_{(i, N)} \in$ $\mathbb{P}_{\bmod (i+N, p)}$ with no active constraint on terminal state $x_{(i, N)}$. Then at the next state $x_{i+1}=x_{(i, 1)}$ the solution $U_{i+1}^{*}\left(x_{i+1}\right)$ is given by $u_{(i+1, k)}^{*}=u_{(i, k+1)}^{*} \forall k \in \mathbb{N}_{0}^{N-2}$ and $u_{(i+1, N-1)}^{*}=K_{\bmod (i+N, p)} x_{(i+1, N-1)}$. Consequently $U_{i}^{*}\left(x_{i}\right)$ is infinite-horizon optimal. By induction this holds recursively.

If terminal constraint sets $\mathbb{T}_{j}$ are closed, convex polyhedra (see Remark 10) then MPC Problem 12 can be written as a convex quadratic programming $(\mathrm{QP})$ problem.

\section{Modeling systems with asynchronous inputs}

In this section a model for systems with asynchronous inputs is proposed. Consider system (1) and denote by $u_{[k] i}$ input channel $k$ at step $i$. Suppose $u_{i}$ is subject to

$u_{[k] i}=u_{[k] i-1} \quad$ if $k \in \hat{\ell}_{j}$

where $\hat{\ell}_{j} \subseteq\left\{\mathbb{N}_{1}^{m_{j}} \cup \emptyset\right\}$ denotes the set of indices of input channels that cannot be updated at step $j$. For all $j \in \mathbb{N}_{0}^{p-1}$ let $\bar{\ell}_{j}:=\mathbb{N}_{1}^{m_{j}} \backslash \hat{\ell}_{j} \subseteq$ $\left\{\mathbb{N}_{1}^{m_{j}} \cup \emptyset\right\}$ and denote by $\bar{m}_{j} \in \mathbb{N}$ and $\hat{m}_{j} \in \mathbb{N}$ the cardinalities of $\bar{l}_{j}$ and $\hat{\ell}_{j}$, respectively. Note that if $\hat{\ell}_{j}=\mathbb{N}_{1}^{m_{j}}$ then $\bar{m}_{j}=0$ (see Remark 1). Let $\bar{u}_{i} \in \mathbb{R}^{\bar{m}_{j}}$ contain inputs that are updated at step $i$, and $\hat{u}_{i} \in \mathbb{R}^{\hat{m}_{j}}$ inputs that remain unchanged from step $i-1$. Further define $\bar{x}_{i}:=\left[x_{i}^{\top}, \hat{u}_{i}^{\top}\right]^{\top} \in \mathbb{R}^{\bar{n}_{j}}$. Then a model of system (1) containing fewer states and inputs than alternative methods (see below) is defined as follows:

$\bar{x}_{i+1}=\left[\begin{array}{cc}A_{j} & {\left[B_{j}\right]_{\left[:, \hat{j}_{j}\right]}} \\ 0 & \alpha_{j}\end{array}\right] \bar{x}_{i}+\left[\begin{array}{c}{\left[B_{j}\right]_{\left[:, \bar{l}_{j}\right]}} \\ \beta_{j}\end{array}\right] \bar{u}_{i}$.

Matrices $\alpha_{j} \in\{0,1\}^{\hat{m}_{\bmod (j+1, p)} \times \hat{m}_{j}}, \beta_{j} \in\{0,1\}^{\hat{m}_{\bmod (j+1, p)} \times m_{j}} \forall j \in \mathbb{N}_{0}^{p-1}$ are permutation matrices, and not explicitly defined here. The re-modeling procedure of system (1) is performed as follows. For all $j \in \mathbb{N}_{0}^{p-1}$ define

$\mathcal{A}_{j}:=\left[A_{j}^{\top}, 0_{\left\{\hat{m}_{\bmod (j+1, p)}^{\top}, n_{j}\right\}}^{\top}\right]^{\top} \in \mathbb{R}^{\left(n_{j}+\hat{m}_{j}\right) \times n_{j}}$

$\mathscr{B}_{j}:=\left[B_{j}^{\top},\left[I_{m_{j}}\right]_{\left[\hat{\ell}_{\bmod (j+1, p):}^{\top}\right]^{\top} \in \mathbb{R}^{\left(n_{j}+\hat{m}_{j}\right) \times m_{j}}}\right.$

$\mathcal{C}_{j}:=\left[I_{m_{j}}\right]_{\left[:, \bar{l}_{j}\right]} \in\{0,1\}^{m_{j} \times \bar{m}_{j}}$

$\mathscr{D}_{j}:=\left[I_{m_{j}}\right]_{\left[:, \hat{\iota}_{j}\right]} \in\{0,1\}^{m_{j} \times \hat{m}_{j}}$

$\mathcal{E}_{j}:=\left[I_{n_{j}}, 0_{\left\{n_{j}, \hat{m}_{j}\right\}}\right] \in\{0,1\}^{n_{j} \times\left(n_{j}+\hat{m}_{j}\right)}$

$\mathcal{F}_{j}:=\left[0_{\left\{\hat{m}_{j}, n_{j}\right\}}, I_{\hat{m}_{j}}\right] \in\{0,1\}^{\hat{m}_{j} \times\left(n_{j}+\hat{m}_{j}\right)}$

such that

$u_{i}=\mathcal{C}_{j} \bar{u}_{i}+\mathscr{D}_{j} \hat{u}_{i}, \quad x_{i}=\mathcal{E}_{j} \bar{x}_{i}, \quad \hat{u}_{i}=\mathscr{F}_{j} \bar{x}_{i}$,

$\bar{x}_{i+1}=\mathcal{A}_{j} x_{i}+\mathscr{B}_{j} u_{i}$.

Substituting (12) into (13) and (4) yields:

$\bar{x}_{i+1}=\left(\mathscr{A}_{j} \mathcal{E}_{j}+\mathscr{B}_{j} \mathscr{D}_{j} \mathcal{F}_{j}\right) \bar{x}_{i}+\left(\mathscr{B}_{j} \mathcal{C}_{j}\right) \bar{u}_{i}$

$\left(E_{j} \mathcal{E}_{j}+G_{j} \mathscr{D}_{j} \mathcal{F}_{j}\right) \bar{x}_{i}+\left(G_{j} \mathcal{C}_{j}\right) \bar{u}_{i} \leq W_{j}$

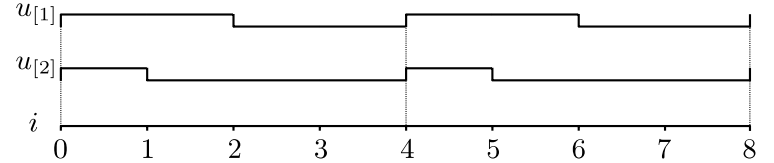

Fig. 1. Asynchronous timing over two periods: $p=4$.

To demonstrate, consider system (1) with $p=4$ and $m_{j}=$ $2 \forall j \in \mathbb{N}_{0}^{3}$. The two input channels are updated according to Fig. 1 . At $j=0$ both input channels, at $j=1$ only $u_{[2]}$, at $j=2$ only $u_{[1]}$, are updated. At $j=3$ neither input channel is updated (see Remark 1 ). The system for $i \in \mathbb{N}_{0}^{4}$ is explicitly stated in Eq. (14). Note that $\left\{\bar{m}_{0}, \bar{m}_{1}, \bar{m}_{2}, \bar{m}_{3}\right\}=\{2,1,1,0\}$ and $\bar{n}_{j}=n_{j}+m_{j}-\bar{m}_{j} \forall j \in \mathbb{N}_{0}^{3}$. Values $\bar{B}_{3}=\emptyset$ and $\bar{u}_{3}=\emptyset$ are notational abuses (see Remark 1 ), but are intended to signify that the system is autonomous when $j=3$.

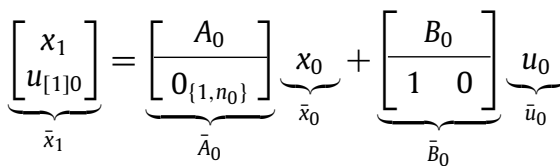

$\left[\begin{array}{c}x_{2} \\ u_{[2] 1}\end{array}\right]=\left[\begin{array}{c|c}A_{1} & {\left[B_{1}\right]_{[:,\{1\}]}}\end{array}\right]\left[\begin{array}{c}x_{1} \\ u_{[1] 0}\end{array}\right]+\left[\begin{array}{c}{\left[B_{1}\right]_{[:,\{2\}]}} \\ 1\end{array}\right] u_{[2] 1}$

$\left[\begin{array}{c}x_{3} \\ u_{[1] 2} \\ u_{[2] 1}\end{array}\right]=\left[\begin{array}{c|c}A_{2} & {\left[B_{2}\right]_{[:,\{2\}]}} \\ \hline 0_{\left\{1, n_{2}\right\}} & 0 \\ 0_{\left\{1, n_{2}\right\}} & 1\end{array}\right]\left[\begin{array}{c}x_{2} \\ u_{[2] 1}\end{array}\right]+\left[\begin{array}{c}{\left[B_{2}\right]_{[:,\{1\}]}} \\ 1 \\ 0\end{array}\right] u_{[1] 2}$

$x_{4}=\left[\begin{array}{ll}A_{3} & B_{3}\end{array}\right]\left[\begin{array}{lll}x_{3}^{\top} & u_{[1] 2} & u_{[2] 1}\end{array}\right]^{\top}+\emptyset \emptyset$.

In Scattolini and Schiavoni (1995) (slightly differently in Colaneri and De Nicolao (1995), Colaneri et al. (1992), Longhi (1994) and Sezer and Šiljak (1990)) system (1) is modeled according to Eq. (15) with $M_{0}:=I_{2}, M_{1}:=\operatorname{diag}(0,1), M_{2}:=$ $\operatorname{diag}(1,0), M_{3}:=0_{\{2,2\}}$ and $\bar{u}_{i}=u_{i}-u_{i-1}$. Note that $\bar{n}_{j}=n_{j}+$ $m_{j}, \bar{m}_{j}=m_{j}=2 \forall j \in \mathbb{N}_{0}^{3}$. Matrices $M_{j}$ force $\left[\bar{B}_{j}\right]_{[:,\{k\}]}$ to zero when input channel $k$ cannot be updated. This causes MPC Problem 12 to contain redundant decision variables, and may result in extra complexity for invariant set methods. In this example the number $v_{j}$ of decision variables is on average twice that when using the model of Eq. (14).

$\bar{A}_{j}=\left[\begin{array}{c|c}A_{j} & B_{j} \\ \hline 0_{\left\{2, n_{j}\right\}} & I_{2}\end{array}\right] \wedge \bar{B}_{j}=\left[\begin{array}{c}B_{j} \\ I_{2}\end{array}\right] M_{j} \quad \forall j \in \mathbb{N}_{0}^{3}$

$\bar{B}_{0}=\left[\begin{array}{c}B_{0} \\ I_{2}\end{array}\right], \quad \bar{B}_{1}=\left[\begin{array}{c}B_{1} \\ I_{2}\end{array}\right]_{[:,\{2\}]}$,

$\bar{B}_{2}=\left[\begin{array}{c}B_{2} \\ I_{2}\end{array}\right]_{[:,\{1\}]}, \quad \bar{B}_{3}=\emptyset$.

In Ling et al. (2005), Richards et al. (2007) and Sågfors et al. (2000) system (1) is modeled by $\bar{A}_{j}$ and $\bar{B}_{j}$ according to Eqs. (15) and (16), respectively. Matrices $\bar{B}_{j}$ of Eq. (16) contain only columns of $\bar{B}_{j}$ in Eq. (15) associated with input channels that can be updated. This avoids the rank-defective $\bar{B}_{j}$ matrices of Eq. (15). However, the models of Eqs. (15) and (16) have larger state dimensions than the model of Eq. (14). Excessive state dimensions may increase the complexity of set invariance methods, invariant sets, and MPC Problem 12.

Consider continuous-time double-integrator $G(s)=s^{-2}$ of Eq. (17), with two input channels updated according to Fig. 1, and sample-period $0.1 \mathrm{~s}$. The following constraints are imposed: $\|x\|_{\infty} \leq 2,\|u\|_{\infty} \leq 1,\left|\left[\begin{array}{ll}1 & 1\end{array}\right] u\right| \leq 1$. The computation-times $\tau$ 
(20 run average) to determine the maximum controlled invariant set $\mathbb{C}^{*}$ of cyclic shift-invariant lifted LTI system (7) using the three different models are tabulated below. Set projection was performed using Fourier elimination. The benefits of using model (14) in this example are clear. Note that no general conclusions can be drawn from this example, as Fourier elimination is sensitive to both problem instance as well as problem size. However, smaller problems frequently result in shorter computation times. This motivational example is used because the plant of Section 6 resulted in excessive computation-times. All computations presented in this paper were performed on a $3.33 \mathrm{GHz}$ processor with Matlab and the Multi-Parametric Toolbox (Kvasnica, Grieder, \& Baotić, 2004).

\begin{tabular}{llllll} 
Model & $\mathbf{n}$ & $\mathbf{m}$ & $\mathbf{r}$ & $\tau[\mathrm{s}]$ & $\tau / \tau^{*}$ \\
\hline Eq. (14) & 12 & 4 & 40 & $\tau^{*}:=32.93$ & 1 \\
Eq. (15) & 16 & 8 & 40 & 121.55 & 3.69 \\
Eq. (16) & 16 & 4 & 40 & 83.79 & 2.54
\end{tabular}

$\dot{x}=\left[\begin{array}{ll}0 & 1 \\ 0 & 0\end{array}\right] x+\left[\begin{array}{ll}0 & 0 \\ 1 & 1\end{array}\right] u$

\section{Multirate nano-positioning system example}

Consider the simple model of a nano-positioning system in Fig. 2. The inputs are base- and piezo-stage forces $f_{b}$ and $f_{p}$. Mass $m_{b}$ is typically a linear motor with large travel, mass $m_{p}$ a piezo element for precision positioning and disturbance rejection, but small travel. The continuous-time LTI dynamics $(n=4, m=2)$ are given by Eq. (18). The following coefficients approximate the atomic force microscope (AFM) of Jones and Goncalves (2010), with a slow, heavy base-stage and a fast, lightly damped piezo-stage: $m_{b}=10 \mathrm{~g}, m_{p}=1 \mathrm{~g}, \delta_{b}=10^{-3} \mathrm{~N} \mathrm{~s} / \mathrm{m}, \delta_{p}=5 \cdot 10^{-6} \mathrm{~N} \mathrm{~s} / \mathrm{m}$, $k=0.25 \mathrm{~N} / \mathrm{m}$. The following constraints are imposed: $\left|x_{b}\right| \leq$ $0.4 \mathrm{~mm},\left|x_{p}\right| \leq 0.4 \mathrm{~mm},\left|x_{b}-x_{p}\right| \leq 0.1 \mathrm{~mm},\left|\dot{x}_{b}\right| \leq 0.03 \mathrm{~m} / \mathrm{s}, \mid \dot{x}_{b}-$ $\dot{x}_{p}|\leq 0.1 \mathrm{~m} / \mathrm{s},| f_{b}|\leq 5 \mathrm{mN},| f_{p} \mid \leq 0.2 \mathrm{mN}$. The system is sampled at $1 \mathrm{kHz}$. Due to the slow base dynamics, input $f_{b}$ is updated only every 10 th sample. This is modeled as system (1) with $p=10, n_{0}=4, m_{0}=2$ and $n_{j}=5, m_{j}=1 \forall j \in \mathbb{N}_{1}^{9}$.

$\ddot{x}_{b}=\frac{1}{m_{b}}\left[-k x_{b}-\left(\delta_{b}+\delta_{p}\right) \dot{x}_{b}+k x_{p}+\delta_{p} \dot{x}_{p}+f_{b}\right]$

$\ddot{x}_{p}=\frac{1}{m_{p}}\left[k x_{b}+\delta_{p} \dot{x}_{b}-k x_{p}-\delta_{p} \dot{x}_{p}+f_{p}\right]$.

The maximum periodic controlled invariant set $\left\{\mathbb{C}_{0}, \ldots, \mathbb{C}_{p-1}\right\}$ was determined using the algorithm of Blanchini and Ukovich (1993) (see Remark 11), requiring 19 iterations and $6.2 \mathrm{~min}(20$ run average). Set projection was performed using Qhull (Barber, Dobkin, \& Huhdanpaa, 1996). The maximum periodic positively invariant set $\left\{\mathbb{P}_{0}, \ldots, \mathbb{P}_{p-1}\right\}$, for system (1) under periodic linear state-feedback $u_{i}=K_{j} x_{i}$ with matrices $K_{j}$ given by Eq. (3), was determined by applying the algorithm of Gilbert and Tan (1991) to cyclic shift-invariant lifted LTI system (7), and unlifting the resulting maximum positively invariant set $\mathbb{P}^{*}$. The algorithm of Gilbert and Tan (1991) required 13 iterations. The entire computation required $27.3 \mathrm{~s}$ (20 run average). Note that the maximum periodic positively invariant set is not employed in this example.

The control objective is reference-tracking of piezo-stage position $x_{p}$. The $x_{p}$ reference trajectory switches between $\pm 0.4 \mathrm{~mm}$ and is known in advance. The state-input reference trajectory $r_{i}$ is determined such that a zero offset achieves minimum-energy

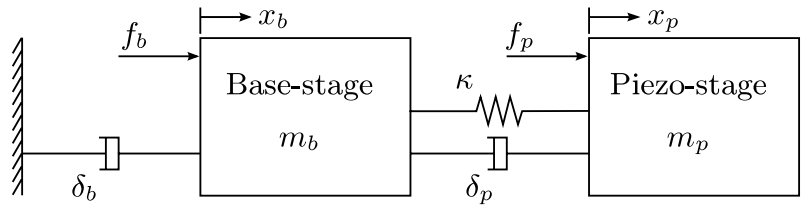

Fig. 2. Nano-positioning system schematic.

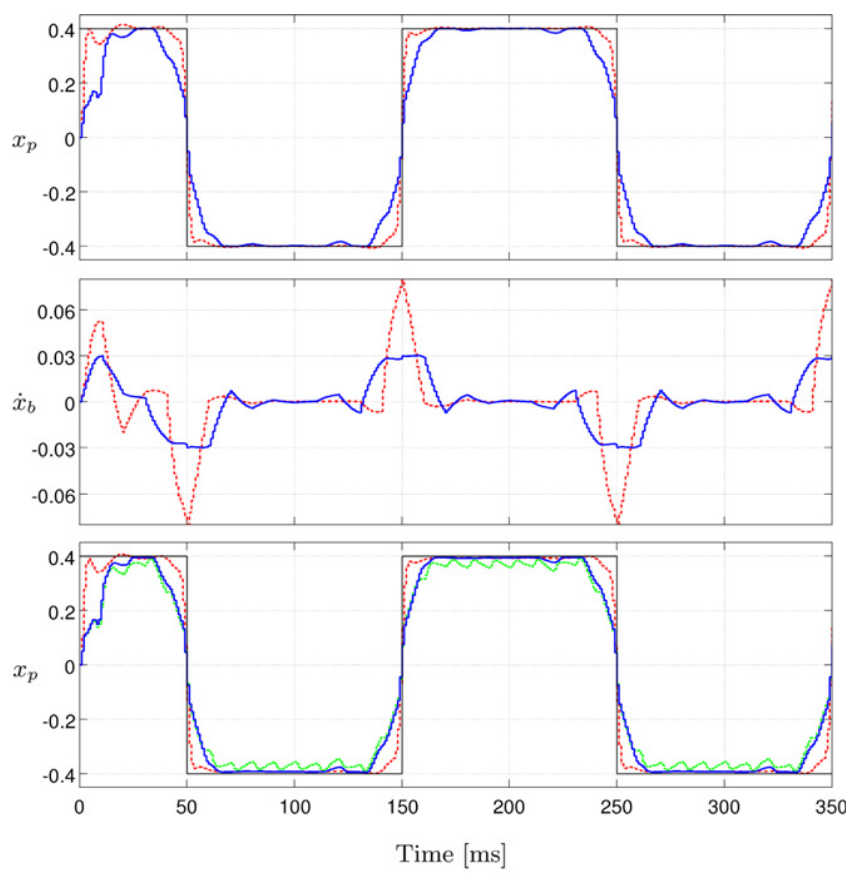

Fig. 3. Top: Piezo-stage position $x_{p}(\mathrm{~mm})$, no mismatch. Middle: Base-stage velocity $\dot{x}_{b}(\mathrm{~m} / \mathrm{s})$, no mismatch. Bottom: Piezo-stage position $x_{p}(\mathrm{~mm})$, with mismatch. Constrained periodic MPC: blue, solid. Unconstrained periodic MPC: red, dash-dotted. Lifted-MPC: green, dashed. Reference: black, solid. Note constraints $\left|x_{p}\right| \leq 0.4,\left|\dot{x}_{b}\right| \leq 0.03$. (For interpretation of the references to color in this figure legend, the reader is referred to the web version of this article.)

steady-state satisfaction of the $x_{p}$ reference to be tracked, for LTI system (18) with synchronous inputs. Reference trajectory $r_{i}$ is known in advance for all $i \in \mathbb{N}$, and so the employed predicted reference values are $r_{(i, k)}=r_{i+k} \forall(i, k) \in \mathbb{N} \times \mathbb{N}_{0}^{N}$ (see Remark 13). Consequently the closed-loop system non-causally responds to reference changes before they occur. Cost matrices $Q$ and $R$ equal to the identity, $S=0$, and a prediction horizon of $50 \mathrm{~ms}(N=50)$ were chosen.

Plotted in Fig. 3 are the piezo-stage position $x_{p}$ (top) and base-stage velocity $\dot{x}_{b}$ (middle). Two control laws are contrasted; constrained periodic MPC (blue, solid) according to Theorem 16, and unconstrained periodic MPC (red, dash-dotted), achieved by solving MPC Problem 12 with $U_{\bmod (i, p)}\left(x_{i}\right)=\mathbb{R}^{v_{\bmod (i, p)}} \forall i \in \mathbb{N}$. In both cases $T_{j}=0 \forall j \in \mathbb{N}_{0}^{p-1}$. The solid black line denotes the $x_{p}$ reference trajectory. Unconstrained control achieves a very fast response at the expense of serious constraint violation in $\dot{x}_{b}$, and a little constraint violation in $x_{p}$. Constrained periodic MPC achieves rigorous constraint satisfaction at the expense of a slightly sluggish response in $x_{p}$.

Next a plant-model mismatch is introduced such that: $x_{i+1}=$ $0.99\left(A x_{i}+B u_{i}\right)$. Plotted in Fig. 3 (bottom) is the piezo-stage position $x_{p}$. Three control laws are contrasted; constrained periodic MPC (blue, solid) as above, unconstrained periodic MPC 
(red, dash-dotted) as above, and Lifted-MPC (LMPC) (green, dashed). In the LMPC approach the linear periodic system is lifted according to Meyer and Burrus (1975) over an entire period and LTI-MPC methods applied. LMPC recomputes the input trajectory after every period, not at every step, and thus suffers the idiosyncrasies of Lee et al. (2001) (similarly Böhm, Raff et al., 2009) as explained in Section 1. The constrained/unconstrained periodic MPC solutions do not track the reference as closely as in the nominal case of Fig. 3(top), but have not changed significantly in any way. The LMPC solution rigorously satisfies the constraints, but produces a larger average error and is highly oscillatory. Oscillations are generally undesirable, and occur because the lifting to an LTI system reduces the effective sample-period to $0.1 \mathrm{kHz}$. Thus the accumulated effects of the plant-model mismatch are only rejected at every 10 th step. With no plant-model mismatch LMPC is nearly identical (thus not plotted) to constrained periodic MPC. This demonstrates how even small disturbances/mismatches may cause severe performance degradation in LMPC, whereas the proposed periodic MPC approach degrades more gracefully with increasing disturbance/mismatch levels.

The periodic MPC control law derivation of Section 4 does not explicitly enforce robustness. However, the plant-model mismatch was chosen such that it does not destroy the strong feasibility property. To see this, recall that the sets of feasible states are convex and contain the origin within their interiors (see Remark 10 and the proof of Theorem 16). In this example the strongly feasible reference-tracking MPC problem formulation and Theorem 16 were employed, because except for Lee et al. (2001), which employ LTI-MPC methods, the other referenced periodic MPC methods cannot handle such situations. Similar periodic control law considerations, not in an MPC context, were made in Blanchini and Ukovich (1993).

\section{Conclusion}

By characterizing suitably general periodic invariant sets, and combining them with well-known results on optimal unconstrained periodic LQR, constrained periodic MPC problems that are least-restrictive and strongly feasible, and constrained periodic MPC problems that yield stabilizing and optimal control laws were designed. Periodic systems with time-dependent dimensions provide a compact and unified modeling framework for synthesizing control laws for systems with asynchronous control inputs. This periodic modeling framework is trivially extensible to modeling asynchronous outputs, e.g. caused by multiple measurement rates (multirate), or when sampling outputs at fixed time-intervals in ordered sequence (multiplexed). Outputs were ignored here because the discussion of control law synthesis focused on state-feedback. The extension of output-feedback MPC theory from LTI to linear periodic systems, and the use of periodic systems to model asynchronous input/output behavior for control of nonlinear systems, are of future research interest.

\section{References}

Barber, C. B., Dobkin, D. P., \& Huhdanpaa, H. (1996). The Quickhull algorithm for convex hulls. ACM Transactions on Mathematical Software, 22(4), 469-483. www.qhull.org.

Bemporad, A., \& Morari, M. (1999). Robust model predictive control: a survey. In Robustness in identification and control: Vol. 245 (pp. 207-226). Springer.

Bittanti, S., \& Colaneri, P. (2000). Invariant representations of discrete-time periodic systems. Automatica, 36(12), 1777-1793.

Bittanti, S., \& Colaneri, P. (2009). Periodic systems filtering and control. Springer.

Blanchini, F. (1999). Set invariance in control. Automatica, 35(11), 1747-1767.

Blanchini, F., \& Ukovich, W. (1993). Linear programming approach to the control of discrete-time periodic systems with uncertain inputs. Journal of Optimization Theory and Applications, 78(3), 523-539.
Böhm, C., Raff, T., Reble, M., \& Allgöwer, F. (2009a). LMI-based model predictive control for linear discrete-time periodic systems. In Lecture notes in control and information sciences: Vol. 384. Springer.

Böhm, C., Yu, S., \& Allgöwer, F. (2009b). Predictive control for constrained discretetime periodic systems using a time-varying terminal region. In Proc. conf. methods and models in automation and robotics, Poland.

Chu, E. K. W., Fan, H. Y., Lin, W. W., \& Wang, C. S. (2004). Structure-preserving algorithms for periodic discrete-time algebraic Riccati equations. International Journal of Control, 77(8), 767-788.

Colaneri, P., \& De Nicolao, G. (1995). Multirate LQG control of continuous-time stochastic systems. Automatica, 31(4), 591-596.

Colaneri, P., \& Longhi, S. (1995). The realization problem for linear periodic systems. Automatica, 31(5), 775-779.

Colaneri, P., Scattolini, R., \& Schiavoni, N. (1992). LQG optimal control of multirate sampled-data systems. IEEE Transactions on Automatic Control, 37(5), 675-682.

Dória, C. E. T., \& Hennet, J. C. (1999). (A, B)-invariant polyhedral sets of linear discrete-time systems. Journal of Optimization Theory and Applications, 103(3), 521-542.

Flamm, D. S. (1991). A new shift-invariant representation for periodic linear systems. Systems \& Control Letters, 17(1), 9-14.

Gilbert, E. G., \& Tan, K. T. (1991). Linear systems with state and control constraints: the theory and application of maximal output admissible sets. IEEE Transactions on Automatic Control, 36(9), 1008-1020.

Gohberg, I., Kaashoek, M. A., \& Lerer, L. (1992). Minimality and realization of discrete time-varying systems. In Time-variant systems and interpolation: Vol. 56 (pp. 261-296). Birkhäuser.

Gondhalekar, R., \& Jones, C. N. (2009). Model predictive control of linear periodic systems-a unified framework including control of multirate and multiplexed systems. In Proc. 48th IEEE CDC, China.

Goulart, P. J., Kerrigan, E. C., \& Maciejowski, J. M. (2006). Optimization over state feedback policies for robust control with constraints. Automatica, 42(4), 523-533.

Jones, C. N., \& Goncalves, J. (2010). A cost-effective atomic force microscope for undergraduate control laboratories. IEEE Transactions on Education, 53(2), $328-334$.

Kern, B., Böhm, C., Findeisen, R., \& Allgöwer, F. (2009). Receding horizon contro for linear periodic time-varying systems subject to input constraints. In Lecture notes in control and information sciences: Vol. 384. Springer

Kim, K. B., Lee, J.-W., \& Kwon, W. H. (2000). Intervalwise receding horizon $H_{\infty}$ tracking control for discrete linear periodic systems. IEEE Transactions on Automatic Control, 45(4), 747-752.

Kvasnica, M., Grieder, P., \& Baotić, M. (2004). Multi-Parametric Toolbox (MPT). http://control.ee.ethz.ch/ mpt/.

Lee, Y. I., \& Kouvaritakis, B. (2006). Constrained robust model predictive control based on periodic invariance. Automatica, 42(12), 2175-2181.

Lee, J. H., Natarajan, S., \& Lee, K. S. (2001). A model-based predictive control approach to repetitive continuous processes with periodic operations. Journal of Process Control, 11(2), 195-207.

Ling, K. V., Maciejowski, J. M., \& Wu, B. F. (2005). Multiplexed model predictive control. In Proc. 16th IFAC world congress, Czech Republic.

Longhi, S. (1994). Structural properties of multirate sampled-data systems. IEEE Transactions on Automatic Control, 39(3), 692-696.

Lovera, M., \& Varga, A. (2005). Optimal discrete-time magnetic attitude control of satellites. In Proc. 16th IFAC world congress, Czech Republic.

Maciejowski, J. M. (2002). Predictive control with constraints. Pearson.

Mayne, D. Q., Rawlings, J. B., Rao, C. V., \& Scokaert, P. O. M. (2000). Constrained model predictive control: stability and optimality. Automatica, 36(6), 789-814.

Meyer, R. A., \& Burrus, C. S. (1975). A unified analysis of multirate and periodically time-varying digital filters. IEEE Transactions on Circuits and Systems, CAS-22(3), 162-168.

Park, B., \& Verriest, E. I. (1989). Canonical forms on discrete linear periodically timevarying systems and a control application. In Proc. 28th IEEE CDC, USA.

Qin, S. J., \& Badgwell, T. A. (2003). A survey of industrial model predictive control technology. Control Engineering Practice, 11(7), 733-764.

Reble, M., Böhm, C., \& Allgöwer, F. (2009). Nonlinear model predictive control for periodic systems using LMIs. In Proc. 10th EUCA ECC, Hungary.

Richards, A., Ling, K. V., \& Maciejowski, J. M. (2007). Robust multiplexed model predictive control. In Proc. 9th EUCA ECC, Greece.

Sågfors, M. F., Toivonen, H. T., \& Lennartson, B. (2000). State-space solution to the periodic multirate $H_{\infty}$ control problem: a lifting approach. IEEE Transactions on Automatic Control, 45(12), 2345-2350.

Scattolini, R., \& Schiavoni, N. (1995). A multirate model-based controller. IEEE Transactions on Automatic Control, 40(6), 1093-1097.

Sezer, M. E., \& Šiljak, D. D. (1990). Decentralized multirate control. IEEE Transactions on Automatic Control, 35(1), 60-65.

Varga, A. (2004). Computation of minimal periodic realizations of transfer-function matrices. IEEE Transactions on Automatic Control, 49(1), 146-149.

Varga, A. (2007). An overview of recent developments in computational methods for periodic systems. In Proc. 3rd IFAC workshop on periodic control systems, Russia.

Varga, A. (2008). On solving periodic Riccati equations. Numerical Linear Algebra with Applications, 15(9), 809-835. 


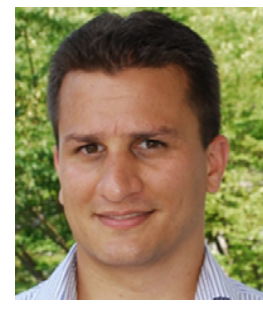

Atomic Energy Authority.
Ravi Gondhalekar was born in Boston, USA, in 1979. He received B.A. and M.Eng. degrees in Engineering in 2002 from the University of Cambridge, UK, and a Ph.D. degree in Informatics in 2008 from the Tokyo Institute of Technology, Japan. He is currently an assistant professor at Osaka University, Japan. His research interests
include model predictive control, constrained control, set nvariance and hybrid systems. Ravi has been employed at the Massachusetts Institute of Technology, the University of Cambridge, Princeton University, Pi Technology, the Rutherford Appleton Laboratory and the United Kingdom

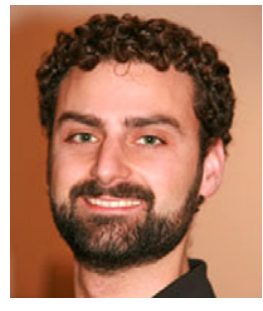

Colin N. Jones is a senior researcher (Oberassistent) at the Automatic Control Laboratory at the ETH in Zurich. He obtained a Ph.D. in 2005 from the University of Cambridge for his work on polyhedral computational methods for constrained control. Prior to that, he was at the University of British Columbia in Canada, where he took a BASc and of British Columbia in Canada, where he took a BASc and
MASc in Electrical Engineering and Mathematics. Colin MASc in Electrical Engineering and Mathematics. Colin control of heating, ventilation and air conditioning to ballistic missile interception. He is co-founder of Apex Optimization; a custom optimization house that focuses on human resource scheduling. His current research interests are in the areas of high-speed predictive control, optimization, energy management and optimal scheduling. 